\title{
Technical Analysis of ZigBee Wireless Communication
}

\author{
Sujan Shrestha, \\ Nepal Open University, \\ Lalitpur, Nepal. \\ ersujan@gmail.com
}

\author{
Subarna Shakya, \\ Pulchowk Campus, \\ Institute of Engineering, \\ Tribhuvan University, \\ Lalitpur, Nepal. \\ drss@ioe.edu.np
}

\begin{abstract}
Information transformed over a long distance without any means like wires, cables and other forms of conductors. The term wireless communication is broad which incorporate different forms of communication and connecting between more devices without any mode of wires through this wireless communication technology. In pre-industrial age, the first network of wireless was developed. This technology has more advanced and featured with upcoming technology and this is one form is future generation. This technology has more impact in growing world. The name wireless communication itself gives the meaning without any physical connection the communication is done in better way. Wireless communication has exponential growth in cellular system and over around two to three billion users.
\end{abstract}

Keywords: - Information transferred, without cable and wires, wireless communication, upcoming technology and future generation.

\section{INTRODUCTION}

Wireless communication is an essential part in different communication devices, this also helps access in remote places. In $19^{\text {th }}$ century, wireless communication was introduced and it was developed more years ago. It actually plays an important role in technology [1]. This medium is used to transfer data over longer distance and without any means of mode like cable and wires or connectors, like the information is transferred in air. In this world of technology, there are more variety of wireless communication like mobile, laptops, and tabs and more. Some of the types of communication of wireless are: GPS, TV, Wi-Fi, Satellite TV, Bluetooth and also more as this technology has more and more upcoming devices in future generation. This wireless communication sends a message, that message is converted into electrical waveform and that is sent through air in faster rate of speed and reaches the destination in baseband signal or message [2]. The transmitter changes baseband signal for transmission. It is transmitted in more subsequent system using a modulator. The channel is used as transmitter where the output is sent based on the type of message. The telegraph was invented first on $1600-1833$, the telegraph from which radio was invented came into existence on 1867-1896 and finally the birth of radio was on 1897-1898. A transatlantic telecommunication is a submarine communication which is a cable that connects one side of Atlantic Ocean, which was invented in the year 1901-1909. Due to the infrastructure of this wireless communication, we can access it remote places at emergency situations. This setup is not that easy but still it is the best choice at many cases. It more cost effective and more accessible with simple infrastructure [3]. The communication is actually split up into three systems: Simple Duplex, Full Duplex and Half Duplex. The services are also provided according to the application we use. Simple Duplex is one-way communication. This communication is directed only in one way. Example: Radio Broadcast System. In Half Duplex, the communication path is double way and it is not simultaneous. Example: Walkie-Talkie. In Full Duplex Communication, here this is like half duplex (i.e,) it's communication is two ways but still it is simultaneous (i.e,) interactions are possible. Example: Mobile Phone [4]. The services of the device may change according to the place of communication (i.e,) the region in which communication takes place is the important factor.

\section{RELATED WORK}

ZigBee communication was built especially for sensor networks and control which uses IEEE standard, which is PAN (Personal Area Network) type of wireless communication. This uses ZigBee alliance product for 
Journal of trends in Computer Science and Smart technology (TCSST) (2020)

Vol.02/ No. 04

Pages: 197-203

https://www.irojournals.com/tesst/

DOI: https://doi.org/10.36548/jtcsst.2020.4.004

communication. This type of standard communication gives physical and also (MAC) Media Access Control layers at very low-data rates and also handles more devices. Some frequencies which operates ZigBee are: 868 $\mathrm{MHz}, 2.4 \mathrm{GHz}$ and $902-928 \mathrm{MHz}$ frequencies. The data rate at which the transmission of data takes place between controllers and sensors is $250 \mathrm{kbps}$ [5]. ZigBee is a mesh network which is deployed with low cost effective and low powered which is used in applications that cover 10-100 meters with range limit. ZigBee is simple and cost effective when compared with ranges short sensor networks like Bluetooth. In present time, none yet has come with sensor and device controller standard communications. ZigBee has high rate standard communication that requires low-latency and low low-energy with low bandwidth [6]. ZigBee has many embedded applications, home automation and also industrial control that is excellent in low-cost and low-power.

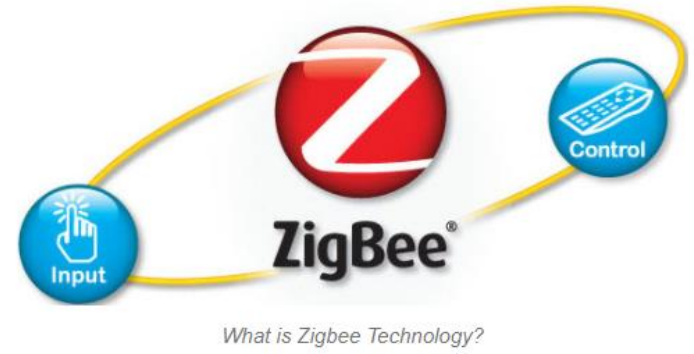

Fig.1 ZigBee Technology

ZigBee has more different configuration and also supports master to master communication or master to slave communication. With power of battery consumption, it is operated in different mode with the results [7]. This network uses router to extend its network connectivity. It allows to interconnection to many nodes in wider area of building network.

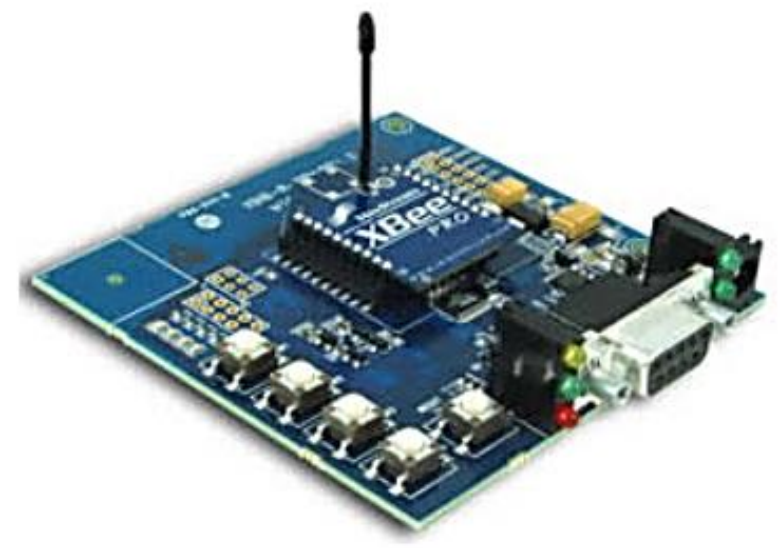

Fig.2 System Structure Device

Three different types of system structure device in ZigBee like Coordinator, Router and End device. There is always a coordinator must in each and every ZigBee network, which plays an important role in routes and bridge of the ZigBee network. Likewise, the coordinator manages information during the transmission of data operations and also while receiving data. The router here acts as intermediate device to permit data through them, they act as an intermediate. The end devices have only few functionalities used to communicate with battery power that is the parent node [8]. Depending on the type of network like star, mesh and tree network, there are many number of coordinators, end devices and routers are used. The ZigBee protocol architecture actually consists on various layer of stack where IEEE is defined by MAC layers and physical layer while protocol is fully completed through accumulator application layers and ZigBee own network. 


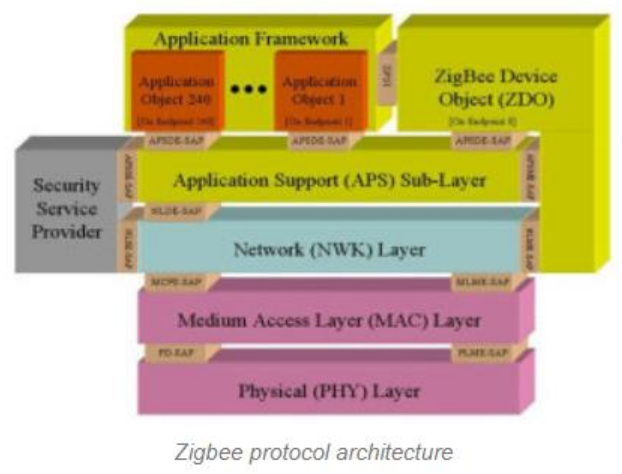

Fig.3 ZigBee Protocol Architecture

Physical layer: Up on transferring or transmitting data and receiving signals, this physical layer does modulation and demodulations. The frequency, number of channels used and data rate are as follows

\begin{tabular}{|ccccc|}
\multicolumn{1}{c}{ BAND } & COVERAGE & DATA RATE & $\begin{array}{c}\text { CHANNEL } \\
\text { NUMBERS }\end{array}$ \\
\hline $2.4 \mathrm{GHz}$ & ISM & Worldwide & $250 \mathrm{khps}$ & $11-26$ \\
\hline $868 \mathrm{MHz}$ & & Europe & $20 \mathrm{klpps}$ & 0 \\
\hline $915 \mathrm{MHz}$ & ISM & Americas & $40 \mathrm{kbps}$ & $1-10$ \\
\hline
\end{tabular}

Physical Layer of Zigbee Protocol

Fig.4 Physical layer construed in ZigBee Protocol

MAC layer: This above layer is responsible for trust worthy transmission of information by access of many network with sense of carrier of Collision Avoidance Multiple Access (CSMA). The beacon frames are transmitted for synchronizing communication.

Network layer: All related operations of network like network setup, connections and disconnection, device configuration, end device, routing and so on...

Support Application Sub-layer: This layer provides all services that is required for ZigBee object devices and application object to interact with network layer in data control services. For matching two devices based on the service provided and needed.

Application Framework: There are two services types are provided like key value and unique message data services. Unique message is actually a developer that is defined structure, where key value is used to get attributes with the application object. An interface is provided by ZDO between APS layer of ZigBee device and application object. Binding, detecting and initiating other devices to network is responsible in this framework.

\section{RESEARCH MOTIVATION AND CHALLENGES}

Operating Modes and topologies of ZigBee: The Two ways of data are transferred in two different modes as: Beacon and Non-Beacon Mode. Beacon mode means, the active state is being monitored continuously by the router and the coordinators and hence power is taken more [9]. Here in this mode, any time any of the node can wake up and transfer data or communicate, as they router and coordinators doesn't sleep. However, overall consumption of power supply requirement is low but power supply is more because many of the devices in network are inactive state for long periods.

In beacon mode, when data communication is not there for end devices, the devices that is the router and coordinator enter the sleep state. This coordinator wakes up periodically and the beacons are transmitted to the router in network [10]. It works with time slot in the beacon network, where the communication needs their results in cycles of lower duty and usage of longer battery where it operates. These two modes of ZigBee that is beacon 
Journal of trends in Computer Science and Smart technology (TCSST) (2020)

Vol.02/ No. 04

Pages: 197-203

https://www.irojournals.com/tcsst/

DOI: https://doi.org/10.36548/jtcsst.2020.4.004

and non-beacon modes manages intermittent (switches of light), sensor data (periodic) and repetitive types of data.

ZigBee Topologies: The most used network configurations and where ZigBee supports many network topologies like star, cluster and mesh tree topologies. One or many coordinators are used in any topologies. In star topology, this network only consists of one coordinator that actually is responsible in managing and initiating devices over network [11]. The remaining devices are called end devices that communicates directly with coordinator. In industries we use all the end point devices that are needed in central controller to communicate, where the topology is simple and easy to destroy.

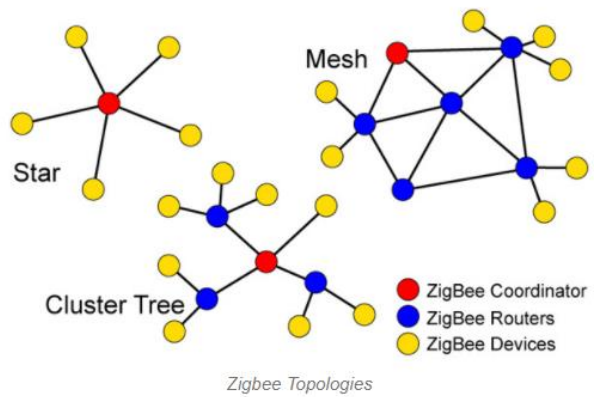

Fig.5 ZigBee Topology

In tree and mesh topologies, there are some extended network in ZigBee where staring them is a responsible taken by coordinators [12]. The device can communicate with any other node of adjacent device where it provides data redundancy as this structure allows. If any node fails, then the topology routers the information automatically to another device. In industries, the main factor is redundancy so here mesh topology is frequently used. In tree cluster network, there is a coordinator with some leaf nodes in each and every cluster and they are connected with coordinators to parent coordinator where it initiates all the network [13]. As ZigBee technology has many advantages like low power for operation node and low cost and its topologies, the applications of several communication range is short and best suited when compared to proprietary communications like $\mathrm{Wi}-\mathrm{Fi}$, Bluetooth and so on...

\section{PROPOSED WORK}

The proposed work comprises that ZigBee communication is better compared with other wireless communications like: Bluetooth and Wi-Fi and so on: ZigBee Communication set the initial setting at the start of the transmission of data, then the data is read in read channel and it verifies from which node the data is being transmitted in Node-ID state [14]. The information is carried with required node channel change if required. If the change is done it create a new channel with the required new change.

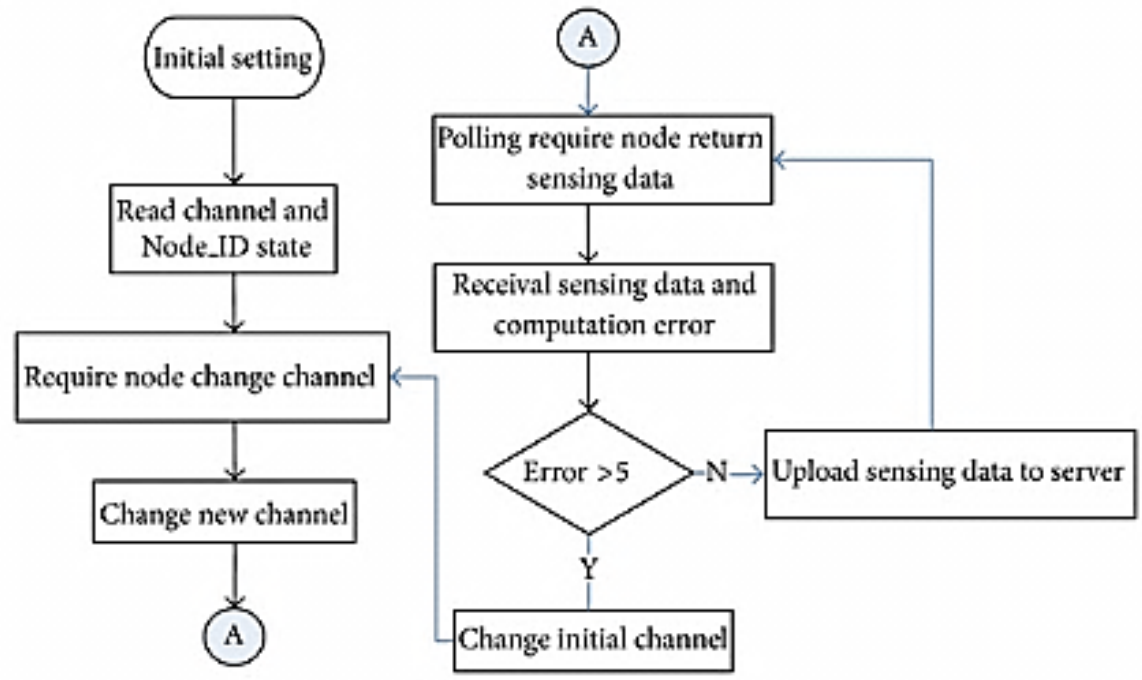

Fig.6 ZigBee Wireless Communication Architecture 
Journal of trends in Computer Science and Smart technology (TCSST) (2020)

Vol.02/ No. 04

Pages: $197-203$

https://www.irojournals.com/tcsst/

DOI: https://doi.org/10.36548/jtcsst.2020.4.004

The data in Wi-Fi has more consumption and data speed takes time and at times occurs at collision, but here in ZigBee data is transmitted at faster rate and also avoidance if data collision is secured, so there is no chance to occur collision [15]. The data is carried with polling that is required node is being checked, verified and data is sent for return of that sensing data. Then the returned data will be sent to the next level of computation and again data is being sent to receive sensing data and the validation of data is done here. And now we go the testing part of the data transmission, here data is validated or tested with a condition that is the error is greater or lesser [16]. Case 1: when data has an error greater than 5 and NO, then data will be uploaded to the sensing data to server. Case 2: when data has an error lesser than 5 and if YES, then data will be sent for change or correction of data with new channel. The data is again sent to start that is to channel change node to create a new channel [17]. Thus the transmission of data takes place with more efficient cost effective way than Wi-Fi communication in ZigBee Communication.

\section{RESULT AND DISCUSSION}

ZigBee communication transfers data to a longer range than $\mathrm{Wi}-\mathrm{Fi}$ at a faster rate without any range limit and the data is sent fast in more secured way. The data is transferred from master to the node 1 and the data is collected faster range and fig.7 shows the throughput of data transmission with graph in fig. 8 shows the speed in which data is sent to node 1 . When data is longer and size its split to network ranges and sent faster, fig.9 shows the division in node that is the network and sent faster rate and that is given in fig. 10 with graph in which data rate in sent at faster speed and increases comparatively.

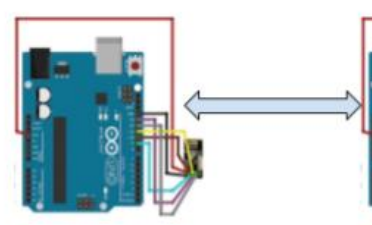

MASTER

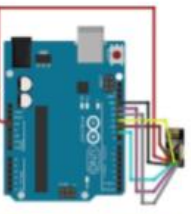

NODE 1

Fig.7 Transmission of data from master to node

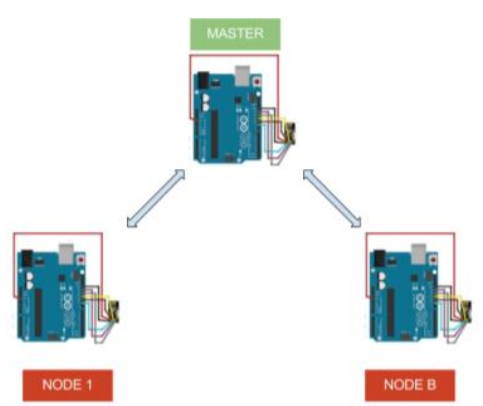

Fig.9 Transmission is split into two noes of network

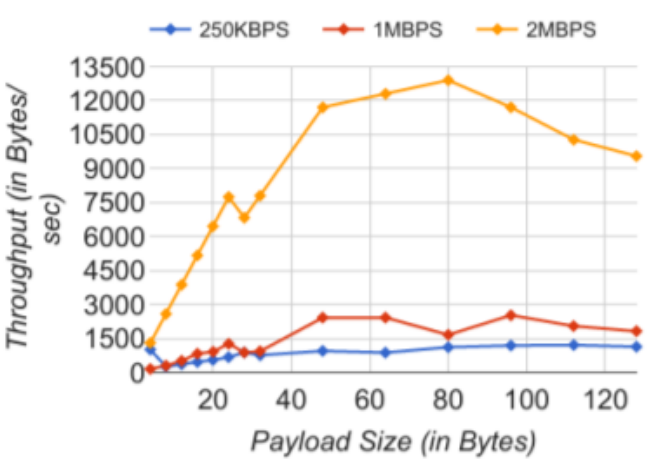

Fig.8 Payload transfer at faster speed

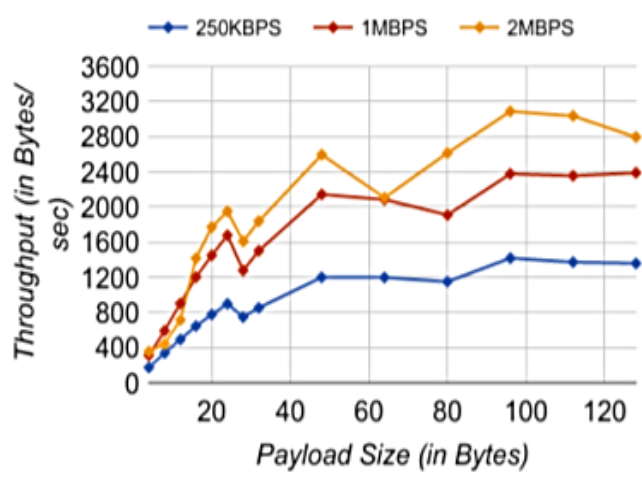

Fig.10 Data size increases with speed 


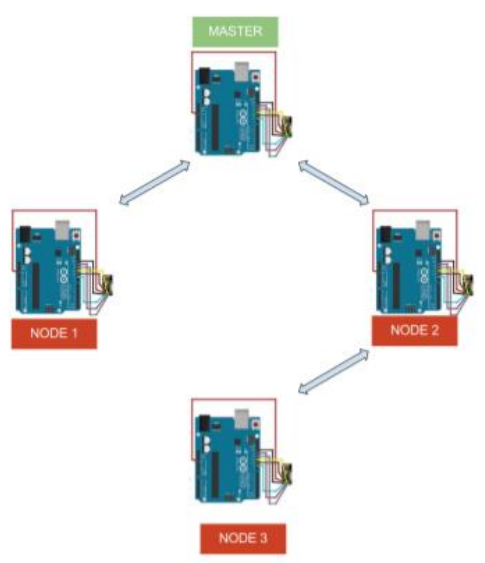

Fig.11 Data nodes are split and transferred

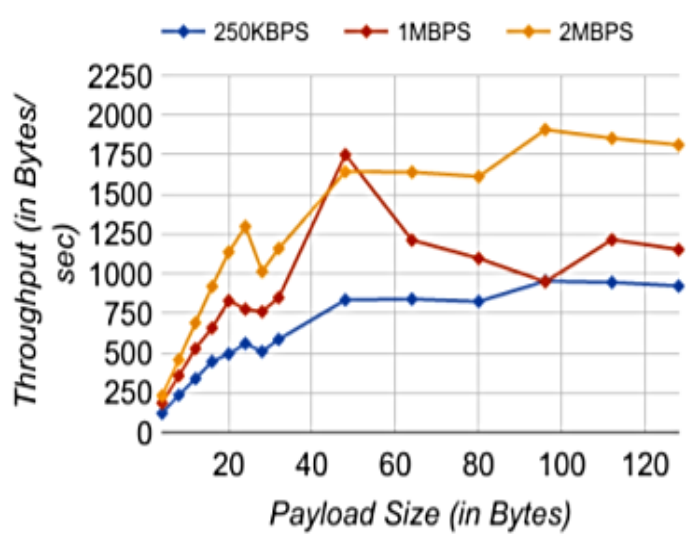

Fig.12 Speed increases with data size

The data is conveyed as payload in these graphs. When data size increases rapidly also in ZigBee the communication is not stopped as this network is more useful in various events and places. So, the network is split and data is transferred in very faster speed rate to reach the destination node. Those data are said as payload and the throughput is the transmission range in which the data is transferred. The above graph shows that ZigBee communication data rapidly increases in the future generation as its more efficient that is ZigBee is low cost effective and low power consumption.

\subsection{Some applications where ZigBee Technology is used:}

Automation: In production and manufacturing industries, a link called communication link is used to monitor continuously in various parameters and critical equipment. And hence the cost of this ZigBee communication is reduced and as well as the control process is optimized for greater reliability.

Home Automation: Home appliances are used remotely which is suited for ZigBee perfectly as lighting control system, control appliance, cooling and heating control system, safety equipment control and surveillance and etc...

Smart Metering: Some smart metering of ZigBee remote operations includes response of energy consumption, support pricing and power theft over security.

Smart Grid Monitoring: Some smart grid monitoring in ZigBee operations that involve temperature monitoring remote like reactive power management and fault locator and so on.

\section{CONCLUSION}

This ZigBee technology is used in wide area network technologies in upcoming and future generations with network that helps to communicate through wireless communication. The broad based development enables standard ZigBee of some reliable wireless technologies with lost cost and solutions and also low complexity that provides an ability for product to go on for years and run in beast way on inexpensive primary batteries.

\section{REFERENCES}

[1] Amram Bengio, E., Senic, D., Taylor, L. W., Tsentalovich, D. E., Chen, P., Holloway, C. L., ... \& Orloff, N. D. (2017). High efficiency carbon nanotube thread antennas. Applied Physics Letters, 111(16), 163109.

[2] Arsalan, M., Ahmad, T. J., \& Noui Mehidi, M. N. (2014, November). Review of downhole wireless communication techniques. In Abu Dhabi International Petroleum Exhibition and Conference. Society of Petroleum Engineers.

[3] Baca, A., Dabnichki, P., Heller, M., \& Kornfeind, P. (2009). Ubiquitous computing in sports: A review and analysis. Journal of Sports Sciences, 27(12), 1335-1346.

[4] Chaitanya, D. E., Sridevi, C. V., \& Rao, G. S. B. (2011, January). Path loss analysis of underwater communication systems. In IEEE Technology Students' Symposium (pp. 65-70). IEEE.

[5] Gabriel, C., Khalighi, M. A., Bourennane, S., Léon, P., \& Rigaud, V. (2013). Monte-Carlo-based channel characterization for underwater optical communication systems. Journal of Optical Communications and Networking, 5(1), 1-12. 
Journal of trends in Computer Science and Smart technology (TCSST) (2020)

Vol.02/ No. 04

Pages: $197-203$

https://www.irojournals.com/tcsst/

DOI: https://doi.org/10.36548/jtcsst.2020.4.004

[6] Goncalves, S. B., Ribeiro, J. F., Silva, A. F., Costa, R. M., \& Correia, J. H. (2017). Design and manufacturing challenges of optogenetic neural interfaces: a review. Journal of neural engineering, 14(4), 041001.

[7] Hashimoto, M., Hata, A., Miyata, T., \& Hirase, H. (2014). Programmable wireless light-emitting diode stimulator for chronic stimulation of optogenetic molecules in freely moving mice. Neurophotonics, 1(1), 011002.

[8] Jeong, J. W., McCall, J. G., Shin, G., Zhang, Y., Al-Hasani, R., Kim, M., ... \& Hong, D. Y. (2015). Wireless optofluidic systems for programmable in vivo pharmacology and optogenetics. Cell, 162(3), 662-674.

[9] Johnson, L., Green, R., \& Leeson, M. (2013, October). A survey of channel models for underwater optical wireless communication. In 2013 2nd International Workshop on Optical Wireless Communications (IWOW) (pp. 1-5). IEEE.

[10] Kaushal, H., \& Kaddoum, G. (2016). Underwater optical wireless communication. IEEE access, 4, 15181547.

[11] Keller, S. D., Zaghloul, A. I., Shanov, V., Schulz, M. J., Mast, D. B., \& Alvarez, N. T. (2014). Electromagnetic simulation and measurement of carbon nanotube thread dipole antennas. IEEE Transactions on Nanotechnology, 13(2), 394-403.

[12] Khalighi, M. A., Gabriel, C., Hamza, T., Bourennane, S., Leon, P., \& Rigaud, V. (2014, July). Underwater wireless optical communication; recent advances and remaining challenges. In 2014 16th International Conference on Transparent Optical Networks (ICTON) (pp. 1-4). IEEE.

[13] Li, B., Huang, J., Zhou, S., Ball, K., Stojanovic, M., Freitag, L., \& Willett, P. (2008, September). Further results on high-rate MIMO-OFDM underwater acoustic communications. In OCEANS 2008 (pp. 1-6). IEEE.

[14] Long, S., \& Miao, F. (2019, December). Research on ZigBee wireless communication technology and its application. In 2019 IEEE 4th Advanced Information Technology, Electronic and Automation Control Conference (IAEAC) (Vol. 1, pp. 1830-1834). IEEE.

[15] Moritz, C. T., Ruther, P., Goering, S., Stett, A., Ball, T., Burgard, W., ... \& Rao, R. P. (2016). New perspectives on neuroengineering and neurotechnologies: NSF-DFG workshop report. IEEE Transactions on Biomedical Engineering, 63(7), 1354-1367.

[16] Shantaram, A., Beyenal, H., Veluchamy, R., \& Lewandowski, Z. (2005). The Challenges of Building Scalable Mobile Underwater Wireless Sensor Networks for Aquatic Applications. Environ. Sci. Technol, 39, 5037-5042.

[17] Shin, G., Gomez, A. M., Al-Hasani, R., Jeong, Y. R., Kim, J., Xie, Z., ... \& Lee, J. L. (2017). Flexible near-field wireless optoelectronics as subdermal implants for broad applications in optogenetics. Neuron, 93(3), 509-521. 